\title{
Metabolismo miocárdico após cardioplegia sangüínea hipotérmica retrógrada contínua
}

\author{
Claudio G. SOBROSA*, Eva JANSSON **, Lennart KAIJSER**, Vollmer BOMFIM*
}

Sobrosa C G, Jansson E, Kaijser L, Bomfim V - Metabolismo miocárdico após cardioplegia sangüínea hipotérmica retrógrada contínua. Rev Bras Cir Cardiovasc 2000; 15 (3): 219-26.

RESUMO: Casuística e Métodos: Realizamos uma análise metabólica da cardioplegia sangüínea hipotérmica retrógrada contínua em um estudo prospectivo de 15 pacientes consecutivos encaminhados para operação eletiva de revascularização miocárdica. Os critérios de inclusão foram doença coronária bi ou triarterial e função ventricular preservada (FE > 40\%). Os critérios de exclusão foram angina instável, diabéticos insulino-dependentes e operações associadas. Três pacientes foram excluídos do trabalho (operação associada e deslocamento do cateter de cardioplegia retrógrada). Amostras de sangue arterial e do seio coronário foram simultaneamente colhidas: antes do início da CEC, na abertura da aorta e com 10, 30 e 60 minutos de reperfusão, para análise do conteúdo de oxigênio e da concentração de lactato. Quatro biópsias miocárdicas foram obtidas do ápice do ventrículo esquerdo: (1) após instalação da CEC (mas antes do pinçamento aórtico), (2) imediatamente após o término da indução cardioplégica, (3) antes do despinçamento aórtico e (4) com 30 minutos de reperfusão, para análise dos níveis de ATP, ADP, AMP e lactato no miocárdio. A isoenzima CK-MB foi analisada no sangue venoso.

Resultados: Não houve mortalidade no grupo. Houve uma diminuição da extração artério-venosa do lactato e do oxigênio pelo coração durante a reperfusão, somente havendo uma recuperação parcial ao final de 60 minutos de reperfusão. O ATP e os outros nucleotídeos tiveram os seus níveis mantidos no miocárdio durante o pinçamento aórtico, mas estes caíram nos primeiros 30 minutos de reperfusão. O lactato acumulouse no músculo cardíaco durante o pinçamento aórtico e diminuiu durante a reperfusão. Houve um aumento da enzima CK-MB, principalmente entre a terceira e sexta horas de pós-operatório.

Conclusões: Do ponto de vista metabólico, o método não evitou o metabolismo anaeróbico durante o período de pinçamento aórtico e que somente com 60 minutos de reperfusão houve uma recuperação metabólica parcial. Essas alterações são, provavelmente, o reflexo da injúria isquêmica celular ocorrida durante o pinçamento aórtico e são de efeito transitório.

DESCRITORES: Miocárdio, metabolismo. Parada cardíaca induzida, métodos. Hipotermia induzida, métodos. Miocárdio, metabolismo. Miocárdio, efeito de drogas. Reperfusão miocárdica. Coração, fisiologia. Revascularização miocárdica.

\section{INTRODUÇÃO}

O grande desenvolvimento da cirurgia cardíaca nos últimos trinta anos se deve muito à reintrodução da cardioplegia cristalóide hiperpotássica no final da década de sessenta. $O$ desafio de realizar cirurgias mais complexas em pacientes mais idosos e mais graves e melhorar as técnicas cirúrgicas e conseqüentemente com tempo de pinçamento aórtico mais prolongado, estimulou muito as pesquisas no campo

\footnotetext{
Trabalho realizado no Hospital Geral de Örebro, Suécia.

Recebido para publicação em agosto de 1999.

* Do Departamento de Cirurgia Cardíaca do Hospital Geral de Örebro, Suécia.

** Do Departamento de Fisiologia Clínica do Hospital de Huddinge, Suécia. Endereço para correspondência: Claudio G. Sobrosa. Hospital Geral Dr. Beda. R. Conselheiro Otaviano, 129 - Centro. Campos dos Goytacazes, RJ, Brasil. CEP 28010-140. Tel. (24) 733-0101. Fax: (24) 723-9690. e-mail: clsobrosa@uol.com.br
} 
da proteção miocárdica. Avanços nas técnicas de proteção miocárdica (1), especialmente com a introdução da cardioplegia sangüínea ${ }^{(2-8)}$, têm sido responsáveis pela menor mortalidade nos pacientes de alto risco. Estudos anteriores têm mostrado melhores resultados clínicos com o uso de cardioplegia sangüínea contínua ${ }^{(9-11)}$ e/ou a perfusão retrógrada através do seio coronário (12-14).

A dificuldade de interpretar pesquisas clínicas reforçou a importância dos trabalhos com a análise metabólica da proteção miocárdica. O objetivo deste estudo é determinar as alterações sofridas pelo miocárdio durante a cardioplegia sangüínea hipotérmica retrógrada contínua.

\section{CASUÍSTICA E MÉTODOS}

\section{Grupo de Pacientes}

Foram analisados 15 pacientes encaminhados eletivamente para revascularização miocárdica e que aceitaram participar deste estudo, o qual foi aprovado pela Comissão de Ética Médica do Hospital. Os critérios de inclusão foram: pacientes com doença coronária obstrutiva bi ou triarterial e função ventricular esquerda preservada (fração de ejeção $>40 \%$ ). Os critérios de exclusão foram: angina instável, diabéticos insulino-dependentes e operações associadas (endarterectomia, aneurismectomia ventricular esquerda, troca de válvula etc). Todos os pacientes foram operados pelo mesmo cirurgião (CGS).

\section{Técnica Operatória}

A conduta anestésica empregada foi a mesma para todos os pacientes. Após a esternotomia mediana e a dissecção da artéria torácica interna esquerda, os pacientes foram heparinizados, o pericárdio aberto e a aorta canulada com uma cânula arterial 22F (DLPâ) e o átrio direito com uma cânula venosa de duplo estágio (DLPâ). A máquina de circulação extracorpórea (CEC) era da marca Stöckertâ e os oxigenadores de membrana eram da marca Didecôa . Foi usada hemodiluição (perfusato de $2000 \mathrm{ml}$ de Ringer lactato) e hipotermia sistêmica com uma temperatura retal entre $28-30^{\circ} \mathrm{C}$.

\section{Cardioplegia}

Um cateter de cardioplegia retrógrada (DLPâ) foi introduzido no seio coronário através de um orifício na parede do átrio direito. A pressão da cardioplegia retrógrada foi continuamente monitorizada e mantida abaixo de $50 \mathrm{mmHg}$. Um pequeno termômetro em forma de agulha foi introduzido no ápice do ventrículo esquerdo, para monitorização contínua da temperatura miocárdica. Um isolador térmico especial foi usado entre o coração e o diafragma, para proteção de possíveis lesões térmicas do nervo frênico e gelo pastoso foi colocado sobre o coração (15). Sangue oxigenado vindo do oxigenador em uma linha em "Y" foi misturado com uma solução de cloreto de potássio na proporção $4: 1$, dando uma concentração sangüínea de potássio de $20 \mathrm{mmol} / \mathrm{l}$ e um hematócrito de $22 \%$. A solução cardioplégica era mantida a uma temperatura de $4-6^{\circ} \mathrm{C}$ e infundida no seio coronário a uma pressão abaixo de $50 \mathrm{mmHg}$. A dose de indução cardioplégica pelo seio coronário foi de 1000 $\mathrm{ml}$ numa velocidade de $200-300 \mathrm{ml} / \mathrm{min}$. Após a indução, a concentração de potássio da cardioplegia foi alterada para $10 \mathrm{mmol} / \mathrm{l}$ (proporção 8:1) e mantida continuamente na velocidade de $50-75 \mathrm{ml} / \mathrm{min}$. Quando necessário, foi usada uma irrigação de solução salina, para facilitar as anastomoses distais, quando o sangramento através das coronárias dificultava a visão das bordas das artérias. O reaquecimento era iniciado durante a última anastomose distal e as anastomoses proximais na aorta eram realizadas com a ajuda de uma pinça arterial colocada tangencialmente na aorta.

\section{Medidas}

A temperatura do ventrículo esquerdo (VE) foi continuamente registrada durante o período de pinçamento aórtico e um computador calculou a Área Tempo-Temperatura (ATT) como um indicador do trauma isquêmico total do coração ${ }^{(16)}$. Amostras de sangue arterial (A) e do seio coronário (SC) foram colhidas para análise do conteúdo de oxigênio e da concentração de lactato. As amostras eram simultaneamente colhidas: antes do início da CEC, na abertura da aorta e com 10, 30 e 60 minutos de reperfusão. Quatro biópsias miocárdicas foram obtidas do ápice do VE com uma pistola especial (Biopty-Cutâ): (1) após instalação da CEC (mas antes do pinçamento aórtico), (2) imediatamente após o término da indução cardioplégica, (3) antes do despinçamento aórtico e (4) com 30 minutos de reperfusão. As biópsias eram imediatamente congeladas em nitrogênio líquido e armazenadas à $-80^{\circ}$ $C$ até a sua análise. No dia da análise as biópsias eram debridadas de gordura, sangue e tecido conectivo sob dissecção microscópica à temperatura $\left(22^{\circ} \mathrm{C}\right)$ e umidade $(30 \%)$ constante e extraídas em $0,5 \mathrm{M}$ de ácido perclórico. $\mathrm{O}$ ácido era removido e neutralizado com $2 \mathrm{M}$ de $\mathrm{KHCO}_{3}$ e analisado por métodos de fluorometria enzimática (17) para determinação das concentrações de trifosfato de adenosina (ATP), difosfato de adenosina (ADP), monofosfato de adenosina (AMP), total de 
Sobrosa C G, Jansson E, Kaijser L, Bomfim V - Metabolismo miocárdico após cardioplegia sangüínea hipotérmica retrógrada contínua. Rev Bras Cir Cardiovasc 2000; 15 (3): 219-26.

nucleotídeos da adenina (TAN) definidos como (ATP+ADP+AMP) e lactato. Nós também calculamos a relação ATP/ADP e a carga energética "energy charge" (EC) definidas como: (ATP + 0,5 ADP)/ (ATP+ADP+AMP). O conceito de carga energética celular (EC) foi proposto por CHAPMAN et al.(18).

A isoenzima CK-MB foi analisada no sangue venoso em amostras retiradas antes da CEC e 1, 3, 6, 912 e 24 horas após o despinçamento aórtico. Um método de espectrometria de massa (IMx STAT CK-MB, Abbot Laboratórios, Abbot Park, IL 60064, USA) que tem alta sensibilidade e sensitividade diagnóstica foi utilizado para determinação da fração MB. As pressões invasivas de artéria radial, átrio direito e esquerdo foram usadas para a monitorização hemodinâmica pós-operatória.

\section{Análise Estatística}

O teste do $t$ de Student foi o método estatístico usado. Diferenças significativas eram definidas como probabilidades de $\mathrm{p}<0,05$ para cada teste. Os valores são apresentados como a média \pm desvio padrão da média.

\section{RESULTADOS}

\section{Resultados Clínicos}

Um paciente foi eliminado do estudo devido à realização de uma endarterectomia, e em 2 pacientes o estudo foi interrompido devido ao deslocamento do cateter do seio coronário. A idade dos pacientes, sexo, fracão de ejeção (FE), número de anastomoses periféricas, tempo de pinçamento aórtico e de CEC estão relacionados na Tabela 1. Não houve mortalidade no grupo. Nenhum paciente apresentou síndrome de baixo débito. Um paciente

TABELA 1

DADOS DOS PACIENTES (MÉDIA \pm DESVIO PADRÃO)

\begin{tabular}{ll}
\hline Número de Pacientes & 12 \\
Homem/Mulher & $10 / 2$ \\
\hline Idade (anos) & $70 \pm 2,3$ \\
FE Pré-op (\%) & $65 \pm 4,1$ \\
Anastomoses Distais (N) & $3,2 \pm 0,2$ \\
\hline Tempo de CEC (min) & $103 \pm 6,7$ \\
Tempo de Pinçamento Ao (min) & $52 \pm 4$ \\
\hline Tempo p/ obter assistolia (sec) & $217 \pm 29$ \\
\hline
\end{tabular}

de 81 anos com uma FE pré-operatória de $45 \%$ necessitou do uso de adrenalina, devido a uma resistência vascular periférica muito baixa com pressão arterial baixa. Ele permaneceu no CTI apenas dois dias. Um paciente foi reoperado devido a sangramento pós-operatório. Quatro pacientes apresentaram fibrilação atrial durante a internação (33\%).

\section{Resultados Metabólicos}

\section{Metabolismo do Lactato}

Medidas seqüenciais do lactato miocárdico extraído (valores negativos indicam produção de lactato) antes da CEC, quando da abertura da aorta e 10, 30 e 60 minutos de reperfusão são mostrados no Gráfico 1. Antes da CEC a diferença arterial-seio coronário (a-sc) de lactato era de $+0,07 \mathrm{mmol} / \mathrm{l}$. No início da reperfusão houve uma mudança para liberação de lactato de $-0,46 \mathrm{mmol} / \mathrm{l}$ que, progressivamente, aumentou para $-1,27 \mathrm{mmol} / \mathrm{l}$ aos 10 minutos de reperfusão. Aos 30 minutos de reperfusão, ainda havia produção de lactato de $-0,3 \mathrm{mmol} / \mathrm{l}$. Apenas aos 60 minutos de reperfusão a diferença artériovenosa de lactato tinha retornado aos níveis iniciais.

\section{Metabolismo do Oxigênio}

Medidas seqüenciais da diferença arterial-seio coronário (a-sc) do conteúdo de oxigênio feitas antes da CEC, quando da abertura da aorta e 10, 30 e 60 minutos de reperfusão são mostrados no Gráfico 2. A diferença a-sc de oxigênio antes da CEC era de $105 \mathrm{ml} / \mathrm{l}$. Quando a aorta foi despinçada, havia uma extração de apenas $30 \mathrm{ml} / \mathrm{l}$. Esta extração baixa aumentou para $75 \mathrm{ml} / \mathrm{l}$ aos 60 minutos de reperfusão, ainda abaixo dos valores iniciais.

\section{GRÁFICO 1}

DIFERENÇA A-V DO LACTATO COLHIDO NA ARTÉRIA RADIAL E NO SEIO CORONÁRIO SIMULTANEAMENTE

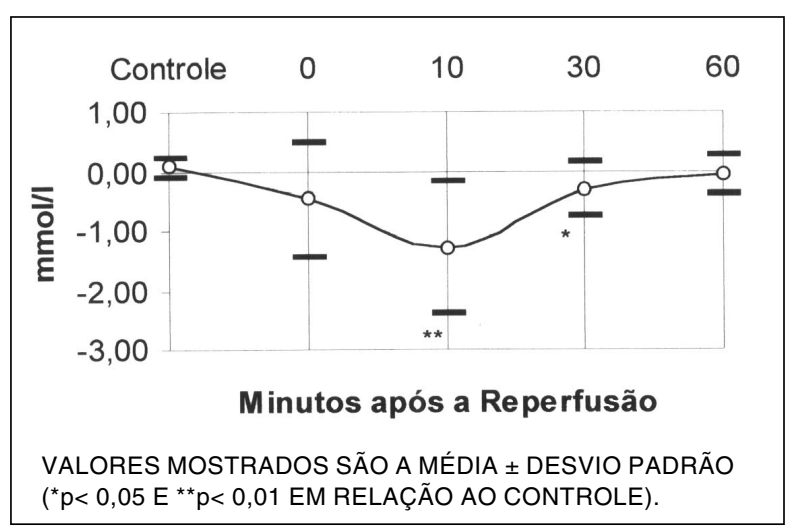


Sobrosa C G, Jansson E, Kaijser L, Bomfim V - Metabolismo miocárdico após cardioplegia sangüínea hipotérmica retrógrada contínua. Rev Bras Cir Cardiovasc 2000; 15 (3): 219-26.

TABELA 2

\begin{tabular}{lccccc}
\hline \multicolumn{5}{c}{ VALORES DO ATP, ADP, AMP, TAN, EC, ATP/ADP ENCONTRADOS NO MIOCÁRDIO (BIÓPSIAS) } \\
\hline & Controle & Ind.Card. & Antes rep & 30'rep & N Válidos \\
\hline ATP & $19,19 \pm 4,1$ & $19,63 \pm 5,4$ & $18,09 \pm 3,2^{*}$ & $16,09 \pm 1,9$ & 12 \\
ADP & $2,82 \pm 0,28$ & $2,70 \pm 0,28$ & $3,99 \pm 3,09^{*}$ & $2,58 \pm 0,47$ & 12 \\
AMP & $0,29 \pm 0,21$ & $0,34 \pm 0,23$ & $0,51 \pm 0,83^{*}$ & $0,17 \pm 0,06^{* *}$ & 12 \\
TAN & $22,25 \pm 3,48$ & $22,72 \pm 5,06$ & $22,60 \pm 0,74$ & $18,86 \pm 1,38$ & 12 \\
EC & $0,92 \pm 0,02$ & $0,91 \pm 0,02$ & $0,88 \pm 0,08^{*}$ & $0,91 \pm 0,01$ & 12 \\
ATP/ADP & $7,07 \pm 1,97$ & $7,36 \pm 2,02$ & $5,04 \pm 2,06^{*}$ & $6,19 \pm 1,37$ & 12 \\
\hline
\end{tabular}

ATP=Trifosfato de Adenosina, $\mathrm{ADP}=$ Difosfato de Adenosina, $\mathrm{AMP}=$ Monofosfato de Adenosina, $\mathrm{TAN}=$ total de nucleotídeos da adenina, $E C=E C, A T P / A D P=$ relação entre o ATP e ADP. Os valores são $\mathrm{mg} / \mathrm{g}$ de músculo seco e apresentados como a média \pm desvio padrão. ( ${ }^{*} p<0,05 e^{* *} p<0,01$ em relação ao controle)

GRÁFICO 2

DIFERENÇA A-V DO DO CONTEÚDO DE OXIGÊNIO COLHIDO NA ARTÉRIA RADIAL E NO SEIO CORONÁRIO SIMULTANEAMENTE

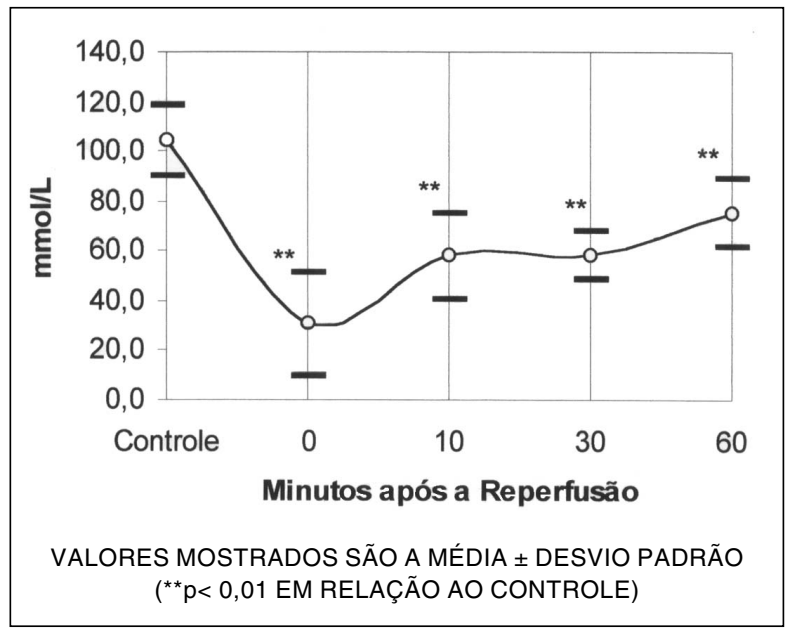

\section{Biópsias Miocárdicas}

A primeira biópsia miocárdica realizada antes do pinçamento aórtico mostra que o ATP no músculo cardíaco era de $19,2 \mathrm{mg} / \mathrm{g}$ de músculo seco. As duas biópsias feitas durante o pinçamento aórtico (logo após a indução cardioplégica e antes do despinçamento aórtico) mostraram níveis de 19,6 e 18,1 mg/g, respectivamente. Aos 30 minutos de reperfusão, houve uma diminuição para 16,1 mg/l. Nós também analisamos a relação ATP/ADP e a EC, que são marcadores da função metabólica celular (Tabela 2).

O controle de lactato no miocárdio antes de CEC foi de $12,75 \mathrm{mg} / \mathrm{g}$ seca de músculo. As duas biópsias realizadas durante o pinçamento aórtico mostraram valores de 18,94 e $32,48 \mathrm{mg} / \mathrm{g}$. Aos 30 minutos de reperfusão, o nível de lactato no músculo havia diminuído para $17,80 \mathrm{mg} / \mathrm{g}$, que não tem significância estatística em comparação com a biópsia de controle (Gráfico 3).

\section{Níveis Plasmáticos da Enzima CK-MB}

O pico de CK-MB ocorreu principalmente 6 horas após o despinçamento aórtico e, em alguns casos, após 3 horas. Os resultados são mostrados na Tabela 3 e no Gráfico 4.

\section{COMENTÁRIOS}

Os resultados clínicos obtidos neste estudo são satisfatórios. Contudo, os achados metabólicos indicam que o método não pode proteger inteiramente o coração do metabolismo anaeróbico e da injúria isquêmica. No entanto, os resultados sugerem que a injúria foi de natureza reversível, provavelmente devido à disfunção celular temporária.

As melhorias nas técnicas de proteção miocárdica têm possibilitado operar-se pacientes com função ventricular esquerda ruim com mortalidade operatória aceitável (19-22). Quando um novo método de proteção miocárdica é clinicamente estudado, é feito, geralmente, em um grupo de pacientes com boa reserva miocárdica e, portanto, torna-se dificil mostrar alguma diferença entre os métodos, pois, normalmente, os resultados são muito similares (23). Isso enfatiza a importância do estudo metabólico para evidenciar diferenças mínimas na disfunção das células miocárdicas. 
Sobrosa C G, Jansson E, Kaijser L, Bomfim V - Metabolismo miocárdico após cardioplegia sangüínea hipotérmica retrógrada contínua. Rev Bras Cir Cardiovasc 2000; 15 (3): 219-26.

TABELA 3

\begin{tabular}{lccccccc}
\hline \multicolumn{7}{c}{ VALORES PLASMÁTICOS DA ENZIMA CKMB } & (MÉDIA \pm DESVIO PADRÃO) \\
\hline Tempo (horas) & Pré-CEC & 1 & 3 & 6 & 9 & 12 & 24 \\
CKMB $(\mathrm{mg} / \mathrm{l})$ & $1.6 \pm 3.9$ & $53 \pm 15$ & $84 \pm 29$ & $97 \pm 37$ & $79 \pm 37$ & $68 \pm 31$ & $24 \pm 13$ \\
\hline
\end{tabular}

GRÁFICO 3

VALORES DO LACTATO ENCONTRADO NO MIOCÁRDIO (BIÓPSIAS)

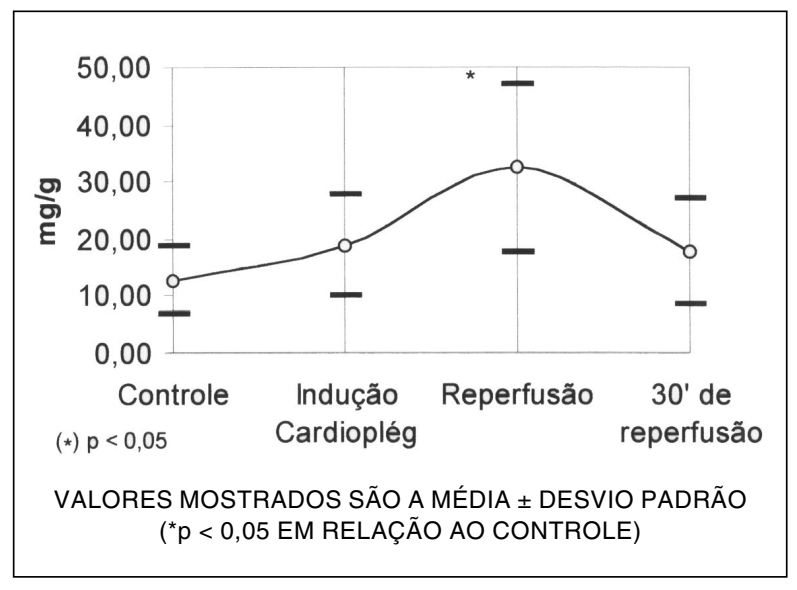

GRÁFICO 4

VALORES DA ENZIMA CK-MB NO SANGUE VENOSO

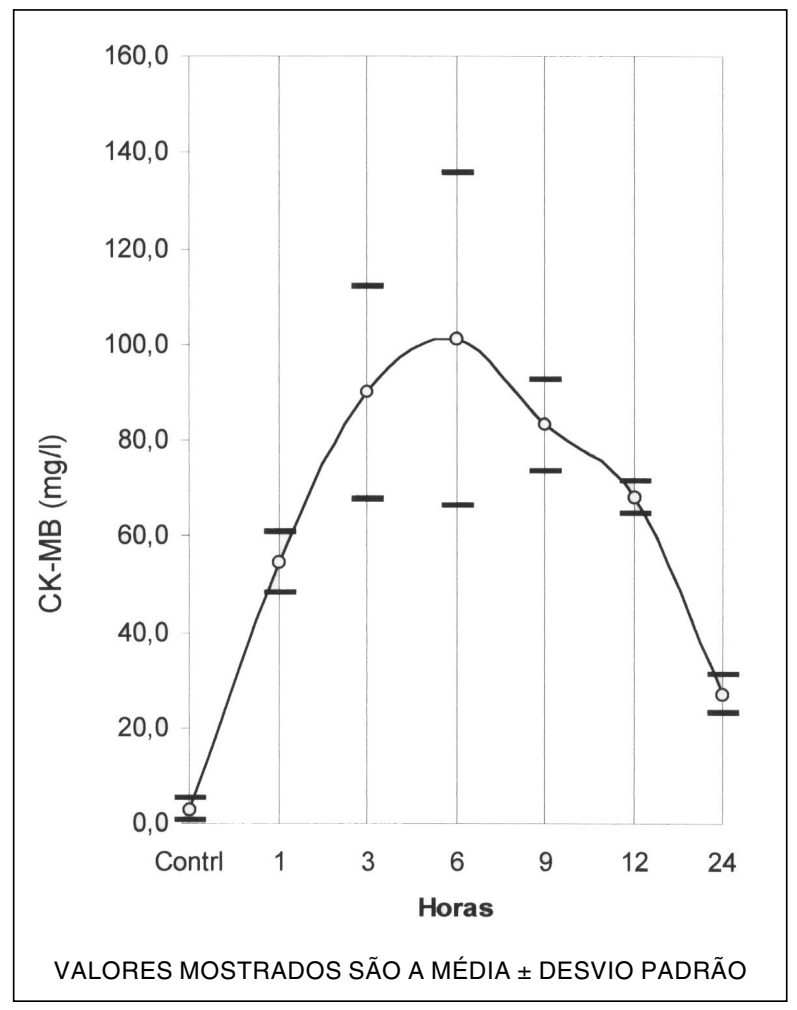

Neste estudo, a diferença arterial-seio coronário de lactato antes da CEC mostrou uma extração normal do lactato. Nós sabemos que o miocárdio, em condições aeróbicas normais, usa o lactato na produção de energia (ATP) e o libera durante o metabolismo aneróbico. Imediatamente após o despinçamento aórtico, havia uma liberação de lactato, o que pode ser derivada da sua produção naquele momento ou de um acúmulo prévio durante o pinçamento aórtico, ou de ambas hipóteses, indicando algum grau de metabolismo anaeróbico ocorrido. Portanto, a cardioplegia sangüínea hipotérmica retrógrada contínua não liberou oxigênio suficiente para as células, provavelmente causada pelo aumento da afinidade da hemoglobina pelo oxigênio (desvio para esquerda na curva de dissociação da hemoglobina) devido à baixa temperatura (24-26) ou devido à presença de regiões do coração não perfundidas satisfatoriamente pela cardioplegia retrógrada. $O$ acúmulo progressivo do lactato no miocárdio durante o tempo de pinçamento aórtico mostrado pelas biópsias reforça o indício da presença de metabolismo anaeróbico, pelo menos na região onde as biópsias foram realizadas. A produção de lactato continuou a aumentar durante os primeiros dez minutos de reperfusão. Este metabolismo anaeróbico, na presença de uma provável liberação de oxigênio, pode indicar a presença de uma disfunção temporária das células miocárdicas $(27,28)$. Somente aos 60 minutos de reperfusão cessou-se a produção de lactato. A extração miocárdica de oxigênio foi muito reduzida após o despinçamento aórtico. Esta extração aumentou progressivamente, durante a reperfusão, embora, após 60 minutos de reperfusão, ainda fosse significantemente menor do que o controle pré-CEC. Isto pode reforçar a hipótese da disfunção metabólica celular, de pelo menos um grupo de células e, provavelmente, ao nível mitocondrial(9,16,23,29-32).

O objetivo dos métodos de proteção miocárdica é a preservação dos níveis dos nucleotídeos da adenina durante o período do pinçamento aórtico(33) e, portanto, minimizar ou evitar a injúria celular. Com as biópsias miocárdicas nós pudemos verificar que o total de ATP miocárdico pode ser mantido durante o período de pinçamento aórtico, mas não durante os primeiros 30 minutos de reperfusão. A relação ADP/ATP e a EC dão uma visão mais dinâ- 
mica do metabolismo celular. Apesar do nível normal de ATP miocárdico no final da oclusão aórtica, a relação ADP/ATP e a EC mostraram uma degradação do ATP. Estes parâmetros metabólicos também indicaram que havia um aumento na produção de ATP com 30 minutos de reperfusão, apesar da diminuição dos seus níveis. Não deve ser esquecido que os níveis de ATP no miocárdio são afetados pela demanda de energia. Como na assistolia, há um consumo muito baixo de ATP, o que pode explicar porque os seus níveis foram mantidos durante o pinçamento aórtico. Durante a reperfusão, o consumo de ATP aumenta com a atividade cardíaca e, apesar do início da recuperação metabólica, a produção aeróbica de ATP não foi suficiente para manter os níveis normais de ATP nos miócitos. Nós sabemos que um coração batendo vazio consome menos $70 \%$ de oxigênio que o coração ejetando (34). Portanto, até com um consumo relativamente baixo, houve uma produção insuficiente de energia (ATP) para evitar o balanço negativo. Este metabolismo celular inadequado durante a reperfusão poderia ser devido a alterações mitocondriais com incapacidade transitória de manter um metabolismo aeróbico normal. Também é possível que a depleção do "pool" dos nucleotídeos da adenina com conseqüente perda dos precursores do ATP retarde a regeneração do "pool" de ATP no miocárdio (35-38).

Nós sabemos que a membrana celular é muito sensível à isquemia e que, após períodos prolongados de anoxia, até moléculas relativamente grandes como as enzimas podem atravessar a membrana celular (16). Os níveis pós-operatórios de CK-MB observados neste grupo de pacientes (Gráfico 4) parece indicar uma função celular temporariamente comprometida.

\section{CONCLUSÕES}

Houve algum grau de metabolismo miocárdico anaeróbico durante o tempo de pinçamento aórtico com a cardioplegia sangüínea hipotérmica retrógrada contínua, apesar da concentração de ATP no miocárdio ser mantida. Contudo, a EC e a relação ATP/ADP, que refletem melhor as alterações na concentração dos nucleotídeos da adenina, parecem indicar um metabolismo catabólico durante este período. Nós observamos profundas alterações no metabolismo da célula miocárdica durante a primeira hora de reperfusão. Estas alterações foram claramente demonstradas pela extração baixa de oxigênio, produção de lactato e produção insuficiente de ATP. Possivelmente, a disfunção da célula miocárdica na reperfusão seja o resultado da injúria celular isquêmica ocorrida durante o pinçamento aórtico que provoca alterações mitocondriais. A lavagem dos precursores do ATP também podem desempenhar um papel importante neste processo. As alterações no metabolismo celular são, provavelmente, transitórias, pois ocorre uma recuperação quase completa após $60 \mathrm{mi}$ nutos de reperfusão.

O retardamento na obtenção de assistolia é uma conhecida desvantagem da cardioplegia retrógrada, e nossos resultados confirmam isto (Tabela 1). Os pacientes tiveram uma boa recuperação pós-operatória, sem mortalidade e apenas um paciente necessitou de suporte inotrópico. Uma desvantagem técnica do método é o sangramento contínuo durante as anastomoses distais, o que pode prolongar um pouco o tempo de pinçamento aórtico (23).

Os resultados clínicos foram satisfatórios e nós concluímos que o método é seguro. Do ponto de vista metabólico, o método não evitou metabolismo anaeróbico durante o pinçamento aórtico. Houve um metabolismo celular inadequado durante a reperfusão, o qual provavelmente seja um reflexo da injúria isquêmica celular ocorrida durante o pinçamento aórtico. Estas alterações metabólicas são transitórias e a recuperação na reperfusão levou 60 minutos. A ineficiência do método de evitar a injúria isquêmica pode ser devido à baixa temperatura da cardioplegia ou à perfusão inadequada pelo uso isolado da cardioplegia retrógrada. Estudos metabólicos com diferentes temperaturas da cardioplegia, o uso de precursores do ATP e a combinação de cardioplegia anterógrada-retrógrada são aconselhados. O problema técnico do sangramento durante as anastomoses distais que ocorre nos métodos de cardioplegia contínua deve ser considerado.

AGRADECIMENTO: Os autores agradecem às enfermeiras Sras. Laila Östensjö, Jenny Larsson, Catharina Hjelm e Jane Strand, pela dedicação aos pacientes deste estudo, assim como, aos anestesistas, perfusionistas e enfermeiras da UTI. 
Sobrosa C G, Jansson E, Kaijser L, Bomfim V - Metabolismo miocárdico após cardioplegia sangüínea hipotérmica retrógrada contínua. Rev Bras Cir Cardiovasc 2000; 15 (3): 219-26.

RBCCV 44205-505

Sobrosa C G, Jansson E, Kaijser L, Bomfim V - Myocardial metabolism after hypothermic retrograde continuous blood cardioplegia. Rev Bras Cir Cardiovasc 2000; 15 (3): 219-26.

ABSTRACT: Material and Methods: An metabolic analyse of hypothermic retrograde continuous blood cardioplegia was done in a prospective study of 15 patients scheduled for elective CABG. Inclusions criteria were double or triple vessel coronary artery disease and preserved left ventricular function (ejection fraction $>40 \%$ ). Exclusions criteria were unstable angina, insulin-treated diabetes mellitus and associated peroperative procedures. Three patients were excluded of the study (associated procedure and coronary sinus catether dislocation). Arterial and coronary sinus blood samples were simultaneouslly taken: before ECC (extracorporeal circulation), when the aortic clamp was takem off and 10,30 and 60 minutes after reperfusion for analysing of oxygen content and lactate concentration. Four transmural left ventricular biopsies samples were obtained: before aortic clamping, immediately after the inicial cardioplegia bolus, immediately before aortic declamping and 30 minutes after reperfusion for analysing of the levels of ATP, ADP, AMP and lactate in the myocardial. The CK-MB isoenzyme was analysed in venous blood samples.

Results: There was no mortality in the group. There was a decrease in the arterial-venous extraction of oxygen and lactate in the heart during reperfusion, occurring a parcial recuperation only at 60 minutes of reperfusion. The ATP and the others nucleotides had their levels in the myocardium maintened during aortic clamping, but these levels decreased during the first 30 minutes of reperfusion. The lactate was accumulated in the heart muscle during aortic clamping and his levels also decreased during reperfusion. The CK-MB levels were elevated specially between the third and sixth post-operative hour.

Conclusions: In the metabolic point of view the method could not avoid an anaerobic metabolism during crossclamping and only after 60 minutes of reperfusion there was a parcial metabolic recuperation. These alterations were probably a reflexion of cellular ischemic injury occurred during cross-clamping and they were of transitory effect.

DESCRIPTORS: Myocardium, metabolism. Heart arrest induced, methods. Hypothermia induced, methods. Myocardium, drug effects. Myocardium reperfusion. Heart physiology. Myocardial revascularization.

\section{REFERÊNCIAS BIBLIOGRÁFICAS}

1 Foker J E, Einzig S, Wang T - Adenosine metabolism and myocardial preservation: consequences of adenosine catabolism on myocardial high-energy compounds and tissue blood flow. J Thorac Cardiovasc Surg 1980; 80: 506-16.

2 Roberts A J, Moran $\mathrm{J} \mathrm{M}$, Sanders $\mathrm{J} \mathrm{H}$ et al. - Clinical evaluation of the relative effectiveness of multidose crystalloid and cold blood potassium cardioplegia in coronary artery bypass graft surgery: a nonrandomized matched-pair analysis. Ann Thorac Surg 1982; 33: 421-33.

3 Cunningham J N, Catinella F P, Spencer F C - Blood cardioplegia experience with prolonged crossclamping. In: Engelman $\mathrm{R} \mathrm{M}$, Levitsk $\mathrm{S}$, eds. $A$ textbook of clinical cardioplegia. Mt. Kisco, New York: Futura, 1982: 242-64.

4 Fabiani J N, Perier P, Chelly J, Camilleri J P, Carpentier A, Dubost $C$. Blood versus crystalloid cardioplegia. In: Engelman R M, Levitsk S, eds. A textbook of clinical cardioplegia. Mt. Kisco, New York: Futura, 1982: 285-96.

5 Catinella F P, Cunningham J N Jr., Adams P X, Snively $S$ L, Gross R I, Spencer F C - Myocardial protection with cold blood potassium cardioplegia during prolonged aortic cross-clamping. Ann Thorac Surg 1982; 33: 228-33.
6 Follette D M, Mulder D G, Maloney J V, Buckberg G D - Advantages of blood cardioplegia over continuous coronary perfusion and intermittent ischemia: experimental and clinical study. J Thorac Cardiovasc Surg 1978; 76: 604-19.

7 Robertson J M, Buckberg GD, Vinten-Johansen J, Leaf J D - Comparison of distribution beyond coronary stenoses of blood and asangueneous cardioplegic solutions. J Thorac Cardiovasc Surg 1983; 86: 80-6.

8 Follete D M, Steed, Foglia R P, Fey, Buckberg G D Advantages of intermittent blood cardioplegia over intermittent ischemia during prolonged hypothermic aortic clamping. Circulation 1978; 58 (Suppl I): 38 [Abstract].

9 Bomfim V, Kaijser L, Bendz R, Sylvén C, Morillo F, Olin C - Myocardial protection during aortic valve replacement: cardiac metabolism and enzyme release following continuous blood cardioplegia. Scand $J$ Thorac Cardiovasc Surg 1981; 15: 141-7.

10 Khuri S F, Warner K G, Josa M et al. - The superiority of continuous cold blood cardioplegia in the metabolic protection of the hypertrophied human heart. $J$ Thorac Cardiovasc Surg 1988; 95: 442-54.

11 Panos A, Christakis G T, Lichtenstein S V, Wittnich C, ElDalati H, Salerno T A - Operation for acute postinfarction mitral insufficiency using continuous oxygenated blood cardioplegia. Ann Thorac Surg 1989; 48: 816-9. 
Sobrosa C G, Jansson E, Kaijser L, Bomfim V - Metabolismo miocárdico após cardioplegia sangüínea hipotérmica retrógrada contínua. Rev Bras Cir Cardiovasc 2000; 15 (3): 219-26.

12 Menasche P, Kural S, Fauchet M et al. - Retrograde coronary sinus perfusion: a safe alternative for ensuring cardioplegic delivery in aortic valve surgery. Ann Thorac Surg 1982; 34: 647-58.

13 Gundry S R \& Kirsh M M - A comparison of retrograde cardioplegia versus anterograde cardioplegia in the presence of coronary artery obstruction. Ann Thorac Surg 1984; 38: 124-7.

14 Partington M T, Acar C, Buckberg G D, Julia P, Kofsky E R, Bugyi H I - Studies of retrograde cardioplegia. I. Capillary blood flow distribution to myocardium supplied by open and occluded arteries. J Thorac Cardiovasc Surg 1989; 97: 605-12.

15 Björk V O, Bomfim V, Olin C - An isolation pad for cold cardioplegia. Scand J Cardiovasc Surg 1978; 12: 177.

16 Bomfim V, Kaijser L, Bendz R, Sylvén C, Olin C Myocardial protection during aortic valve replacement: cardiac metabolism and enzyme release following hypothermic cardioplegia. Scand J Cardiovasc Surg 1980; 14: 43-9.

17 Lowry O H \& Passouneau J V - A flexible system of enzymatic analysis. New York: Academic Press, 1973.

18 Chapman A G, Fall L, Atkinson D E - Adenylate energy charge in Escherichia coli during growth and starvation. J Bacteriol 1971; 108: 1072-86.

19 Teoh K H, Christakis G T, Weisel R D et al. - Increased risk of urgent revascularization. $J$ Thorac Cardiovasc Surg 1987; 93: 291-9.

20 Christakis G T, Weisel R D, David T E, Salerno T A, Ivanov $\mathrm{J}$ - Predictors of operative survival after valve replacement. Circulation 1988; 78 (3 Pt 2): I25-34.

21 Christakis G T, Fremes S E, Weisel R D et al. - Reducing the risk of urgent revascularization for unstable angina: a randomized clinical trial. $J$ Vasc Surg 1986; 3: $764-72$.

Yau T M, Weisel R D, Mickle D A et al. - Optimal delivery of blood cardioplegia. Circulation 1991; 84 (5 Suppl III): $380-8$

23 Engelman R M, Rousou J H, Lemeshow S, Dobbs W A - The metabolic consequences of blood and crystalloid cardioplegia. Circulation 1981; 64 (2 Pt 2): II67-74.

24 Magovern J G Jr., Flaherty J T, Gott VL, Bulkley B H, Gardner T J - Failure of blood cardioplegia to protect myocardium at lower temperatures. Circulation 1962; 66 (2 Pt 2): 160-7.

25 Barner H B - Blood cardioplegia: a review and comparison with crystalloid cardioplegia. Ann Thorac Surg 1991 52: 1354-67.

Lazar H L, Buckberg G D, Manganaro A J et al. - Reversal of ischemic damage with secondary blood cardioplegia. J Thorac Cardiovasc Surg 1979; 78: 688-97.
27 Lazar H L, Foglia R P, Manganaro A J, Buckberg G D - Detrimental effects of premature use of inotropic drugs to discontinue cardiopulmonary bypass. Surg Forum 1978; 29: 276-8.

28 Kane J J, Murphy M L, Bisset J K, de Soyza N, Doherty J E, Straub K D - Mitochondrial function, oxygen extraction, epicardial S-T segment changes and tritiated digoxin distribution after reperfusion of ischemic myocardium. Am J Cardiol 1975; 36: 218-24.

29 Böök K, Kaijser L, Sylvén C, Bomfim V - Normothermic ischaemic arrest in one-vessel coronary artery surgery. Scand J Thorac Cardiovasc Surg 1982; 16: 245-8.

30 Buttner E E, Karp R B, Reves J G et al.- A randomized comparison of crystalloid and blood-containing cardioplegic solutions in 60 patients. Circulation 1984; 69: $973-82$

31 Rosenkranz E R, Okamoto F, Buckberg G D et al.Studies of controlled reperfusion after ischemia. II. Biochemical studies: failure of tissue adenosine triphosphate levels to predict recovery of contractile function after controlled reperfusion. J Thorac Cardiovasc Surg 1986; 92 (3 Pt 2): 488-501.

32 Bomfim V - Continuous blood cardioplegia: a unique approach. In: Engelman R M \& Levitsk S, eds. $A$ textbook of clinical cardioplegia. Mt. Kisco, New York: Futura, 1982: 265-76.

33 Buckberg G D, Brazier J R, Nelson R L, Goldstein S $\mathrm{M}$, McConnell D H, Cooper $\mathrm{N}$ - Studies of the effects of hypothermia on regional myocardial blood flow and metabolism during cardiopulmonary bypass. I. The adequately perfused beating, fibrillating, and arrested heart. J Thorac Cardiovasc Surg 1977; 73: $87-94$

34 Rosenkranz E R, Okamoto F, Buckberg G D, VintenJohansen J, Robertson J M, Bugyi H - Safety of prolonged aortic clamping with blood cardioplegia. II. Glutamate enrichment in energy-depleted hearts. $J$ Thorac Cardiovasc Surg 1984; 88: 402-10.

35 Ely S W, Mentzer R M Jr., Lasley R D, Lee B K, Berne $R M$ - Functional and metabolic evidence of enhanced myocardial tolerance to ischemia and reperfusion with adenosine. J Thorac Cardiovasc Surg 1985; 90: 549-56.

36 Bolling S F, Bies L E, Gallagher K P, Bove E L - Enhanced myocardial protection with adenosine. Ann Thorac Surg 1989; 47: 809-15.

37 Bolling S F, Bies L E, Bove E L, Gallagher K P Augmenting intracellular adenosine improves myocardial recovery. J Thorac Cardiovasc Surg 1990; 99: 469-74.

38 Fiore A C, Naunheim K S, Kaiser G C et al. - Coronary sinus versus aortic root perfusion with blood cardioplegia in elective myocardial revascularization. Ann Thorac Surg 1989; 47: 684-8. 